\title{
因HAD
}

DOI: http://doi.org/10.22585/hospdomic.v5i1.121

\section{Kinesiología en las unidades de hospitalización domiciliaria en Chile}

\section{Kinesiology in hospital at home units in Chile}

\author{
Viviana Marín-Navarro' (i) 0000-0001-7597-932X \\ Francisco Freire Figueroa' (1) 0000-0002-2261-5271 \\ Christian Poblete Figueroa' \\ Roberto Montt Garrido² \\ 1. Unidad de Hospitalización domiciliaria, Complejo asistencial Dr. Sótero del Río, Servicio de Salud Metropolitano Sur Orien- \\ te, Provincia Cordillera, Santiago de Chile. \\ 2. Servicio Nacional de Pesca y Acuicultura, Provincia de Valparaíso. Valparaíso de Chile
}

Correspondencia/Correspondence

Viviana Marin Navarro

Viviana.emn@gmail.com

Recibido/Received

01.09 .2020

Aceptado/Accepted

14.11 .2020

Financiación

Confirmo que este trabajo no obtenido ningún tipo de financiación.
Conflicto de Intereses/Competing interest

Tres de los/las autores/as son kinesiólogos/as que trabajan en una unidad de hospitalización domiciliara en Santiago de Chile.

Contribuciones de autoría/Author contributions La autora y los autores han contribuido por igual en la realiczación de este trabajo.

\section{Agradecimientos/Acknowledgments}

Agradecemos a todos los kinesiólogos/as que confiaron en esta iniciativa y aportaron con los datos presentados. Esperamos que esta investigación contribuya al desarrollo de la kinesiología en esta área, dando a conocer la situación actual, su modo de atención y la caracterización de los kinesiólogos/ as en la unidad de hospitalización domiciliaria en Chile.

CÓMO CITAR ESTE TRABAJO | HOW TO CITE THIS PAPER

Marín-Navarro V, Freire F, Poblete C, Montt R. Kinesiología en las unidades de hospitalización domiciliaria en Chile. Hosp Domic. 2021;5(1):17-28. 


\section{RESUMEN}

Introducción: La hospitalización domiciliaria nace con el objetivo de ser una alternativa a la hospitalización tradicional, similar en recursos y complejidad. Dentro de las prestaciones que se puede entregar, se encuentra la kinesiología. Sin embargo, no existen información específica sobre el perfil ni el quehacer de estos profesionales en dichas unidades. Por lo que se busca con este trabajo, describir y caracterizar el trabajo de los kinesiólogos/as que se desempeñan en unidades de hospitalización domiciliaria en hospitales públicos de Chile.

Materiales y Método: Estudio descriptivo transversal realizado mediante una encuesta a kinesiólogos/as que trabajan en unidades de hospitalización a domicilio en hospitales públicos de Chile. Realizada entre marzo y agosto de 2019. Para el análisis estadístico se utilizó el software Rstudio.

Resultados: De 82 hospitales encuestados, 59 cuentan con hospitalización domiciliaria, de estos, 45 cuentan con kinesiología. Dentro de las áreas más desarrolladas en adultos, son la kinesiología respiratoria (95,3\%), la kinesiología motora $(93,4 \%)$, y la neurorrehabilitación $(90,1 \%)$. En pediatría es la kinesiología respiratoria $(28,1 \%)$. El $32 \%$ de las unidades cuenta con un coordinador/a de profesión kinesiólogo/a.

Conclusiones: La kinesiología permite entregar diferentes prestaciones en domicilio tanto a adultos como niños. Sin embargo, esto surge según la necesidad de cada hospital y no está definido a nivel nacional, por lo que se propone avanzar hacia lineamientos que permitan la estandarizar la incorporación de esta profesión a todas las unidades de hospitalización domiciliaria.

Palabras clave: Servicios de Atención de Salud a Domicilio; Servicios de Atención a Domicilio Provisto por Hospital; Fisioterapia; Modalidades de Fisioterapia.

\section{ABSTRACT}

Introduction: Home hospitalization was created with the aim of being an alternative to traditional hospitalization, similar in resources and complexity. Among the attentions that can be delivered, is kinesiology. However, there is no specific information on the profile or work of these professionals in these units. Therefore, this work seeks to describe and characterize the work of kinesiologists who work in home hospitalization units in public hospitals in Chile.

Materials and Method: Cross-sectional descriptive study carried out by means of a survey of kinesiologists who work in home hospitalization units in public hospitals in Chile between March and August 2019. The Rstudio software was used for the statistical analysis.

Results: Of 82 hospitals surveyed, 59 have home hospitalization, of these, 45 have kinesiology. Among the most developed areas in adults are respiratory kinesiology (95.3\%), motor kinesiology (93.4\%), and neurorehabilitation (90.1\%). In pediatrics it is respiratory kinesiology (28.1\%). $32 \%$ of the units have a coordinator by profession kinesiologist.

Conclusions: Kinesiology allows delivering different benefits at home to both adults and children. However, this arises according to the need of each hospital and is not defined at the national level, so it is proposed to move towards guidelines that allow the standardization of the incorporation of this profession to all home hospitalization units.

Keywords: Home Care Services; Home Care Services, Hospital-Based; Physical Therapy Specialty; Physical Therapy Modalities. 


\section{INTRODUCCIÓN}

\section{Hospitalización domiciliaria}

Las primeras prestaciones hospitalarias a domicilio surgen en el año 1947 en el Hospital Bronx, Nueva York en Estados Unidos, con el concepto de "Home Health Care" como una extensión de los servicios otorgados en el hospital hacia la casa del paciente, con el principal objetivo de descongestionar las salas del hospital y brindar a los pacientes un ambiente más humano y favorable en su recuperación ${ }^{(1-4)}$. Esta modalidad de atención ha sido replicada en varios países en el mundo, así como también en Latinoamérica en países como Colombia, Argentina y Chile.

Se define la hospitalización domiciliaria (HD) como el «traslado al hogar del paciente, el personal, los servicios y la tecnología necesaria para su recuperación o tratamiento, propiciando la participación del paciente y su familia en el proceso terapéutico»(2). Busca ser una «modalidad asistencial alternativa a la hospitalización tradicional en donde el usuario recibe los mismos cuidados que en el hospital, tanto en calidad como en cantidad y sin los cuales habría sido necesaria su permanencia en el hospital» ${ }^{(3,5)}$.

Es importante, además, diferenciar la hospitalización domiciliaria de la atención domiciliaria. Siendo la primera, una modalidad de atención que depende del hospital con atención similar en recursos y complejidad para procesos agudos pero estables, acotados en el tiempo. A diferencia de la atención domiciliaria que depende de la atención primaria y entrega cuidados a largo plazo, habitualmente en pacientes crónicos ${ }^{(3,6)}$. Aunque muchas veces estos dos términos se utilizan indistintamente en la literatura.

\section{Beneficios y desventajas de la hospitalización domiciliaria}

Diversos autores han publicado experiencias positivas con respecto a la hospitalización domiciliaria tanto en estudios controlados y no controlados, en los países donde se ha implementado. Demostrando múltiples beneficios, tanto para el centro asistencial en relación a la reducción del costo económico en las estancias hospitalarias ${ }^{(2,7,8)}$ y a la optimización del recurso cama ${ }^{(3)}$, como también beneficios directos para el paciente, tales como el recibir una atención más humanizada, aumentando su comodidad y bienestar al encontrarse en su propio hogar, mejorando la participación y la responsabilidad del paciente con respecto a su propia salud, haciendo partícipe de este proceso también a la familia, entre otras $\operatorname{cosas}^{(1,6,9,10)}$.

Sin embargo, hay cosas que han sido pesquisadas de las cuales no existe mayor documentación, de cómo en algunos casos se extiende el periodo de la hospitalización, la posible sobrecarga al cuidador al requerir mayores cuidados, y los costos de traslados de los profesionales al domicilio, siendo esto último difícil de calcular de manera global, ya que la distancia y características geográficas no es igual en todas las regiones.

\section{Hospitalización domiciliaria en Chile}

En Chile, el uso de camas hospitalarias corresponde a un costo significativo de recursos destinados a la salud pública ${ }^{(3)}$, debido a su alta demanda. Es por ello, que se buscan y nacen alternativas a la hospitalización tradicional que son de bajo costo, como lo es el hospital de día y las unidades de hospitalización domiciliaria (UHD). En los últimos 20 años, han existido diversos intentos de establecer hospitalizaciones domiciliarias a lo largo del país, las cuales aparecen y desaparecen de forma intermitente según los recursos disponibles y las iniciativas de profesionales de la salud 
que se encuentran en un lugar y momento determinado. Finalmente, este modelo se implementa de manera formal a través del Ministerio de Salud (MINSAL) en diez hospitales públicos desde el año $2011^{(3)}$, Ilegando a ser alrededor de 24 hospitalizaciones domiciliarias en el año 2014(11) y unas 59 al momento de recolección de datos.

\section{Kinesiología en hospitalización domiciliaria}

A pesar de los años que llevan funcionando las unidades de hospitalización domiciliaria en Chile y alrededor del mundo, hay poca información sobre el perfil, la integración, el rol y las intervenciones que realiza el kinesiólogo (nombre que se le da al fisioterapeuta en nuestro país) en dichas unidades $^{(11)}$. Sin embargo, hay registro de experiencias positivas en relación a las labores que el kinesiólogo puede y realiza en domicilio, como la titulación de oxigenoterapia, el manejo de ventilación mecánica no invasiva (VMNI), el manejo de pacientes con traqueostomía ${ }^{(9,10-13)}$, la rehabilitación posterior a un accidente cerebro vascular ${ }^{(14,15)}$ y la rehabilitación posterior a una cirugía de prótesis de cadera(16).

\section{MÉTODOS}

Se realizó un estudio descriptivo transversal. Los datos fueron recolectados a partir de kinesiólogos/ as que se desempeñan en UHD de todos los hospitales públicos a nivel nacional. Se consultó inicialmente vía telefónica para saber si el hospital contaba con UHD y si contaba con kinesiología, en caso de ser positiva ambas respuestas, se enviaba una encuesta vía correo electrónico que debían contestar todos los kinesiólogos/as que trabajaran en dicha unidad. Esta encuesta, se aplicó entre los meses de marzo y agosto del año 2019, la cual fue elaborada por los autores del estudio y contaba de 18 preguntas de selección múltiple con opción de libre respuesta para casos excepcionales. Se evaluaron características de los profesionales, características laborales y distintas funciones que cumplen en su quehacer diario en las UHD.

El análisis estadístico se llevó a cabo con el software Rstudio, y Microsoft Excel 2013 para la elaboración de los gráficos.

Este estudio fue aprobado por el comité de ética del servicio de salud metropolitano sur oriente (SSMSO) y se resguardaron las consideraciones éticas y de confidencialidad mediante la aplicación de un consentimiento informado.

Cabe destacar que estos datos fueron resultados de una muestra auto gestionada y no representan datos oficiales del Ministerio de Salud.

\section{RESULTADOS}

\section{Caracterización de la Muestra}

De un total de 82 hospitales de mediana y alta complejidad registrados a lo largo del país, el $72 \%(\mathrm{~N}=59)$ cuentan con UHD, de ellos el 76,2\% ( $N=45)$ cuenta con kinesiología dentro de sus prestaciones; de estos últimos, el 69\% (N=31) respondieron la encuesta vía correo electrónico. En la tabla 1 se puede apreciar el detalle por región. 
Tabla 1. Hospitales con unidades de hospitalización domiciliaria en Chile

\begin{tabular}{|c|c|c|c|c|}
\hline Región & $\begin{array}{l}N^{\circ} \text { de } \\
\text { Hospitales }\end{array}$ & $\begin{array}{l}N^{\circ} \text { de } \\
\text { Hospitales con } \\
\text { UHD }\end{array}$ & 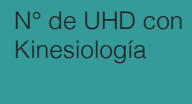 & $\begin{array}{l}\text { Hospitales que } \\
\text { respondieron }\end{array}$ \\
\hline XV Región de Arica y Parinacota & 1 & 1 & 1 & 0 \\
\hline I Región de Tarapacá & 1 & 1 & 1 & 1 \\
\hline II Región de Antofagasta & 3 & 2 & 1 & 0 \\
\hline III Región de Atacama & 2 & 1 & 1 & 1 \\
\hline IV Región de Coquimbo & 4 & 3 & 2 & 2 \\
\hline V Región de Valparaíso & 9 & 6 & 5 & 2 \\
\hline $\begin{array}{l}\text { VI Región del Libertador General } \\
\text { Bernardo O'Higgins }\end{array}$ & 4 & 4 & 4 & 3 \\
\hline VII Región del Maule & 6 & 3 & 3 & 3 \\
\hline XVI Región de Ñuble & 2 & 1 & 1 & 1 \\
\hline VIII Región del Biobío & 9 & 5 & 3 & 3 \\
\hline IX Región de la Araucanía & 8 & 5 & 3 & 2 \\
\hline XIV Región de los Ríos & 1 & 1 & 1 & 1 \\
\hline X Región de Los Lagos & 4 & 3 & 2 & 2 \\
\hline $\begin{array}{l}\text { XI Región de Aysén del General } \\
\text { Carlos Ibáñez del Campo }\end{array}$ & 2 & 2 & 1 & 1 \\
\hline $\begin{array}{l}\text { XII Región de Magallanes y de la } \\
\text { Antártica Chilena }\end{array}$ & 2 & 1 & 0 & 0 \\
\hline $\begin{array}{l}\text { Región Metropolitana de } \\
\text { Santiago }\end{array}$ & 24 & 20 & 16 & 9 \\
\hline TOTAL & 82 & 59 & 45 & 31 \\
\hline
\end{tabular}

Un total de 64 kinesiólogos/as respondieron la encuesta, de los cuales el 47\% ( $N=30)$ pertenecía al género femenino y el 53\% ( $\mathrm{N}=34)$ al género masculino. De los 64 encuestados, dos personas no entregaron información respecto a su edad, tomando esto en consideración, el promedio 
de edad fue de 29,6 años $( \pm 3,44)$, siendo el rango de edad más predominante el de 25-27 años $(\mathrm{N}=26)$, seguido del rango de 31-33 años $(\mathrm{N}=18)$, y la categoría entre 37-39 años la de menor representatividad $(\mathrm{N}=1)$. Del total de los encuestados, el $68 \%(\mathrm{~N}=50)$ tenía formación de post título (cursos y diplomados) mientras que el $8 \%(\mathrm{~N}=5)$ contaba con post grado de magister en áreas relacionadas a la kinesiología.

En cuanto a la experiencia laboral previa a trabajar en la UHD, un $18,8 \%(\mathrm{~N}=12)$ se desempeñaba en el nivel de atención primario, un $15,6 \%(\mathrm{~N}=10)$ en el nivel secundario y un $23,4 \%(\mathrm{~N}=15)$ proviene del nivel terciario. Un $18,8 \%(\mathrm{~N}=12)$ proviene del sector privado de salud y un $21,9 \%(\mathrm{~N}=14)$ no contaba con experiencia laboral clínica previa.

En cuanto a la antigüedad de los kinesiólogos/as que se desempeñan en las UHD. El 31,2\% ( $N=21)$ lo hacía desde hace $1-2$ años, el 32,8\% ( $N=20)$ desde hace 3-5 años, el 20,3\% ( $N=10)$ de 6 o más y un $15,6 \%(N=13)$ desde hace menos de un año.

La calidad contractual de los kinesiólogos es variable. Un 44\% ( $N=28)$ trabaja en modalidad de contrata, el $48 \%(\mathrm{~N}=31)$ a honorarios (entrega de servicios de forma independiente por un tiempo limitado), y el $5 \%(\mathrm{~N}=3)$ señalan pertenecer a una entidad privada que presta servicios al sistema público. El número de kinesiólogos/as en cada unidad también es variable, según las necesidades de cada centro. Un 45\% ( $\mathrm{N}=29)$ de las unidades cuenta con 1 a 2 kinesiólogos/as, el 21\% (N=14) de 5 a 6 kinesiólogos/as, un 13\% ( $N=8)$ cuenta con 3 a 4, mismo porcentaje para las unidades que cuentan con más de 9 kinesiólogos/as, y un 8\% ( $\mathrm{N}=5)$ cuenta con 7 a 8. Es importante mencionar que se consideraron solo los profesionales que trabajan de forma anual y no aquellos que trabajan por algunos meses en específico en forma de reemplazo o refuerzo. El detalle puede ser visualizado en la tabla 2.

Tabla 2. Número de kinesiólogos que trabajan en las unidades de HD

\begin{tabular}{|l|l|l|}
\hline$N^{\circ}$ de Kinesiólogos & $N^{\circ}$ de UHD & Porcentaje \\
\hline 1 a 2 & 29 & $45 \%$ \\
\hline 3 a 4 & 8 & $13 \%$ \\
\hline 5 a 6 & 14 & $21 \%$ \\
\hline 7 a 8 & 5 & $8 \%$ \\
\hline$>9$ & 8 & $13 \%$ \\
\hline
\end{tabular}

Dentro de las unidades con mayor número de kinesiólogos/as, un 32\% ( $N=20)$ cuentan con coordinación de kinesiología independiente del resto de los estamentos de la unidad, el 29\% ( $N=9$ ) tiene como coordinación a un enfermero/a y el 39\% ( $\mathrm{N}=25)$ por un médico. En relación con la modalidad de trabajo, un $54 \%(\mathrm{~N}=35)$ se desempeña en un sistema de 44 horas semanales en horario diurno (de lunes a viernes), un 33\% ( $\mathrm{N}=21)$ en modalidad cuarto turno modificado (dos días de 12 horas trabajo y dos días de descanso) y un $13 \%(\mathrm{~N}=8)$ se encuentra con menos de 44 horas de trabajo en horario diurno. El $80 \%$ ( $N=51)$ cumple una función clínica/asistencial, un 14\% ( $N=9)$ una función 
clínica/administrativa, un 5\% ( $\mathrm{N}=3)$ tiene una función de coordinación tiempo completo y solo el $1,5 \%(N=1)$ realiza funciones de jefatura de la UHD.

En cuanto a la seguridad de realizar labores fuera del ambiente hospitalario, el $78 \%(N=54)$ señala no tener ningún seguro de accidente laboral especifico por desempeñarse en $\mathrm{HD}$, el $19 \%$ $(\mathrm{N}=12)$ reconoce contar con un seguro laboral asociado al centro hospitalario, y el 3\% $(\mathrm{N}=2)$ señala no saber.

\section{Áreas de la kinesiología}

Los kinesiólogos/as abarcan distintas áreas tanto en pacientes adultos como pacientes pediátricos. En el área de adultos, el 95,3\% (N=62) de los encuestados señalaron realizar intervenciones relacionadas a la kinesiología respiratoria (KTR), el 93,4\% ( $N=61)$ a la kinesiología motora (KTM), un $90,1 \%(\mathrm{~N}=59)$ a la neurorrehabilitación (NRHB), un 59,4\% ( $\mathrm{N}=39$ ) a cuidados paliativos (CP), un $9,4 \%(N=6)$ a rehabilitación oncológica y un 3,1\% $(N=2)$ a la gestión y administración.

En el área pediátrica solo el 28,1\% ( $N=19)$ realiza $K T R$, el 10,9\% $(N=10)$ realiza NRHB y el 6,3\% $(\mathrm{N}=5)$ realiza intervenciones relacionadas a la kinesiología motora (figura 1).

Figura 1. Áreas de la kinesiología desarrolladas en las unidades de HD

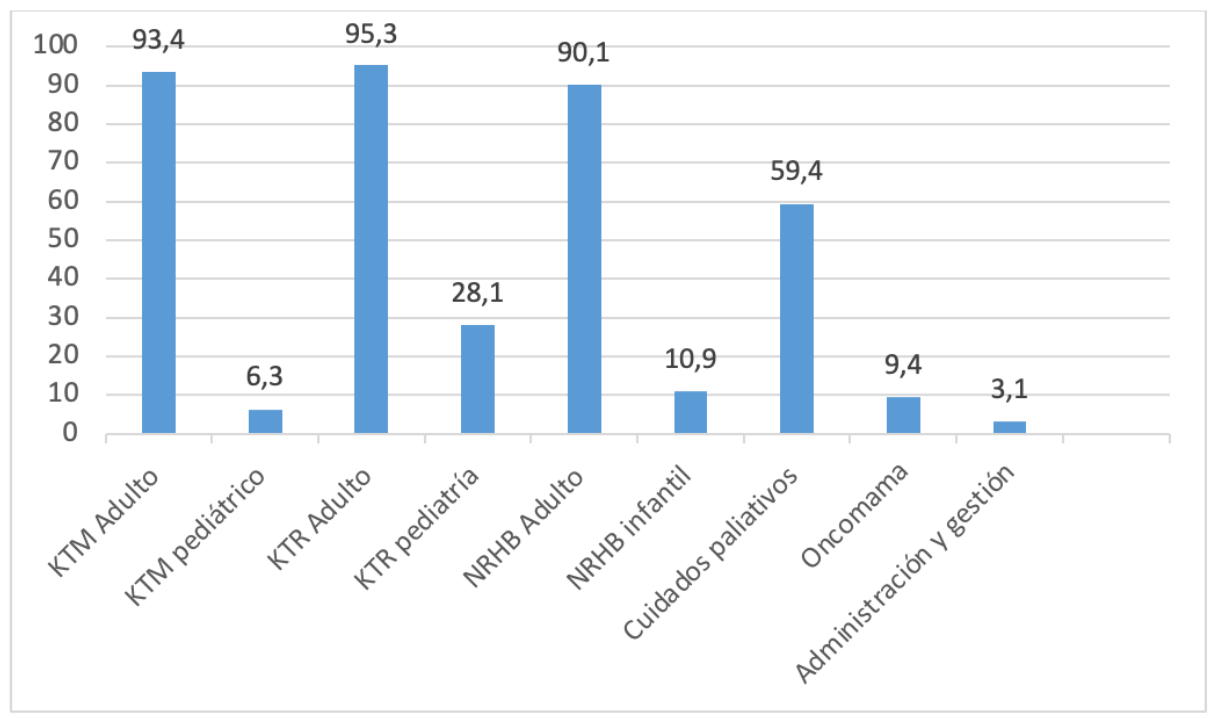

\section{Función Asistencial}

La función asistencial se define como los procedimientos que se realizan de manera específica en cada una de las distintas áreas de desempeño. De manera general, el 89\% ( $\mathrm{N}=57$ ) realiza control de signos vitales y el $96 \%(\mathrm{~N}=62)$ realiza registro en fichas clínicas. Con relación a la función educativa, el 98\% ( $N=63$ ) realiza educación en el domicilio y un 75\% ( $N=48)$ realiza educación intrahospitalaria previo al ingreso; un 30\% (N=9) realiza capacitaciones en distintas temáticas rela- 
cionadas a la $\mathrm{HD}$ al resto de los estamentos y un $8 \%(\mathrm{~N}=5)$ realiza labores de docencia a profesionales en formación.

Un 16\% (N=10) realiza rehabilitación oncológica basada principalmente en la prevención de linfedema en pacientes mastectomizadas (oncomama). Un 88\% (N=56) realiza neuro rehabilitación en pacientes adultos y pediátricos y un 7,8\% $(\mathrm{N}=5)$ señaló realizar intervenciones en relación con la estimulación temprana.

En relación al área respiratoria (figura 2) un 96\% ( $N=62$ ) señaló realizar técnicas kinésicas respiratorias, uso y educación en inhalo terapia de dosis medida (IDM) y aspiración de secreciones, un 95\% ( $\mathrm{N}=61)$ manifestó haber realizado intervenciones en oxigenoterapia tanto en indicación, titulación y educación, un $87 \%(\mathrm{~N}=56)$ indicó haber realizado toma de muestras para cultivo de secreciones, un $84 \%(\mathrm{~N}=54)$ intervino en manejo de paciente con traqueostomía, un 39\% $(\mathrm{N}=25)$ ejecutó nebulizaciones, un 38\% ( $N=24)$ desempeñó actividades en relación al manejo de ventilación mecánica no invasiva, un 1,6\% (N=2) realizó manejo de cánula de alto flujo.

Figura 2. Procedimientos realizados con relación a la kinesioterapia respiratoria

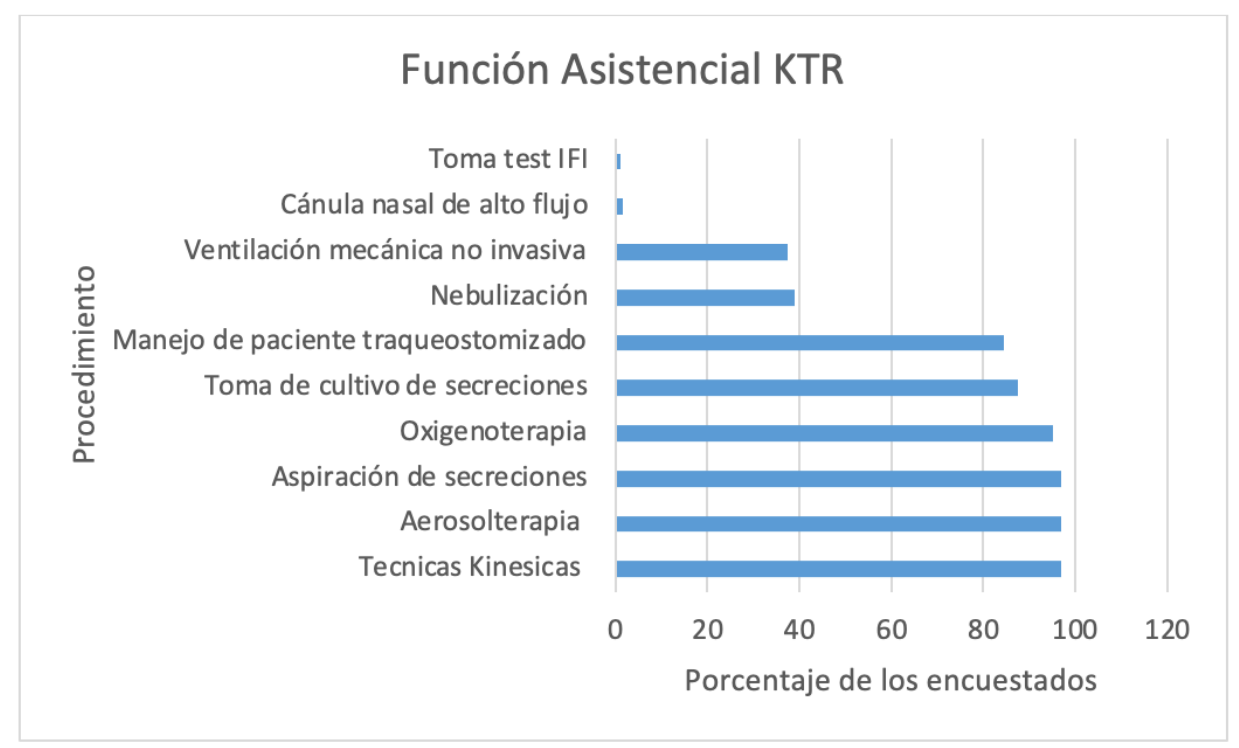

En el área de la kinesiología motora (figura 3), el 91\% ( $N=58)$ realiza manejo de pacientes con dependencia severa (paciente postrado), el 89\% ( $N=57)$ desempeña funciones en rehabilitación post quirúrgica (post- qx), el 78\% ( $\mathrm{N}=51$ ) participa en la prevención de lesiones por presión (LPP), el 73\% ( $N=47)$ realiza rehabilitación traumatológica no quirúrgica y un 39,1\% (N=25) efectúa manejo de órtesis. 
Figura 3. Procedimientos realizados con relación a la kinesioterapia motora

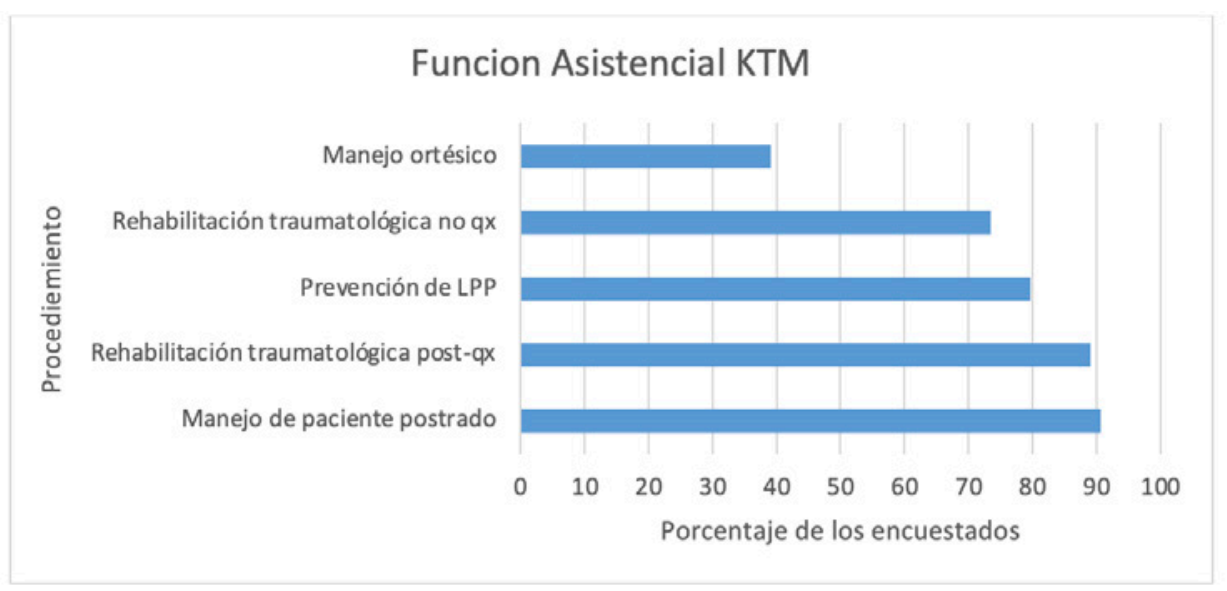

Con respecto a la gestión, el 84\% ( $N=53$ ) señala tener tareas de planificación de las visitas a los pacientes y gestión de reuniones con el resto del equipo, el 70\% ( N=44) participa en la elaboración de registros estadísticos, el 63\% ( $N=40)$ gestiona solicitudes de insumos, el 62\% ( $N=39)$ realiza evaluación de pacientes en sala (intrahospitalarios) previo al ingreso a la UHD, el 51\% ( $N=32)$ participa en la coordinación de distintas prestaciones con otras unidades de apoyo, el 48\% (N=30) participa en la elaboración de protocolos interno, un 46\% ( $\mathrm{N}=29)$ realiza notificación de eventos adversos, el 35\% ( $N=22)$ efectúa categorización de usuarios, un 19\% ( $N=12)$ desarrolla actividades de investigación, el 11\% ( $N=7)$ coordina gestiones con la unidad de gestión de cama y un $6 \%(N=$ 4) realiza gestión y educación de ayudas técnicas.

\section{DISCUSIÓN}

La UHD se posiciona cada vez más como una alternativa para optimizar los recursos sanitarios disponibles, evitando saturar los hospitales con pacientes que pueden recibir atención en su propio domicilio ${ }^{(7)}$. Especialmente en lugares críticos como las unidades de urgencia ${ }^{(17)}$. Siendo el kinesiólogo/a un actor principal en este objetivo.

La encuesta fue respondida por un $69 \%$ de las unidades de hospitalización domiciliaria de distintas regiones, logrando obtener información variada y representativa de nuestro país.

Las unidades en su gran mayoría tienen poca antigüedad, y las más antiguas del país no superan los 12 años dando a conocer lo joven que es este tipo de atención en Chile. La edad promedio de los kinesiólogos que trabajan en HD es más bien joven siendo el rango de edad predominante entre los 25-29 años quienes en su mayoría tienen capacitaciones de post título (cursos y diplomados) pero muy pocos con post grado (8\%) y no en temas específicos de HD, ya que en Chile no existen. La mayoría cuenta con experiencia previa en el sistema público de salud en sus distintos niveles dando a conocer, de una u otra manera, el compromiso por la salud pública. Hecho importante para poder entender el objetivo general de las HD y las necesidades de los pacientes atendidos. 
Uno de los puntos débiles investigados es la modalidad de contratos que mantienen, dado que un gran porcentaje de profesionales se desempeña en calidad contractual de honorarios, manteniendose en esa condición desde 1 a 5 años, contando con poca estabilidad laboral debido a esto.

Con relación al número de kinesiólogos/as, las unidades más antiguas y que pertenecen en su mayoría a hospitales de alta complejidad con mayor número de prestaciones, cuentan hasta con 11 kinesiólogos/as con una coordinación independiente de otros estamentos, contribuyendo así a la autonomía total del quehacer kinésico. Esto contrasta de manera importante con la mayoría de los encuestados, quienes señalan depender de una coordinación médica o de enfermería. Es en este punto se hace visible la necesidad de que el área de kinesiología cuente con una coordinación independiente del resto de los estamentos, entendiendo que son los propios kinesiólogos quienes debiesen decidir la cantidad, frecuencia y tipos de terapias a realizar en los pacientes. Se propone así, que las unidades que cuenten con más de 4 kinesiólogos/as cuenten con una coordinación de la misma profesión. Esto se sustenta con el trabajo de las HD más antiguas donde en la gran mayoría cuentan con coordinación de kinesiología.

La modalidad de trabajo es muy variable, ya que depende de las necesidades propias de cada hospital. La mayoría de los entrevistados desempeñan sus funciones en modalidad de horario diurno de lunes a viernes y solo un 33\% en modalidad de turno cubriendo así los fines de semana, entendiendo que el objetivo de las unidades de hospitalización domiciliaria es entregar un servicio similar al del hospital, se hace necesario contar con jornadas laborales de 12 horas y en sistema de turnos para poder abarcar los 7 días de la semana, pensando que en este contexto la atención nocturna se hace compleja y más aún cuando la seguridad en el ámbito extra hospitalario no está garantizada. Con respecto al tipo de pacientes, es principalmente adultos ya que no todas las HDs nivel nacional cuentan con atención pediátrica. La hospitalización de pacientes pediátricos requiere de una especialización tanto de profesionales como de los elementos técnicos que implican la atención. Generalmente una vez afiatado el trabajo con pacientes adultos, las unidades estarían en condiciones de abrirse a ingresos pediátricos, y es la lógica que han seguido las unidades de mayor antigüedad. Sin embargo, aunque el número de ingresos pediátricos es menor en una mirada global, en temporada de invierno este aumenta considerablemente ${ }^{(10)}$ especialmente de aquellos pacientes con enfermedades crónicas ${ }^{(18)}$.

Dentro de la función asistencial, la labor más desarrollada es la clínico/asistencial y la menos desarrollada es la de docencia, pudiendo ser la cantidad de móviles y la seguridad, el principal impedimento para recibir a estudiantes dentro de estas unidades. La principal área de desarrollo corresponde a la kinesiología respiratoria, seguida por la kinesiología motora y por último la intervención kinésica en el área de la neuro rehabilitación. En pacientes pediátricos predomina la intervención kinésica en patologías respiratorias. También hay otras intervenciones que se realizan en menor porcentaje en ciertos establecimientos y que pudieran replicarse fácilmente, como son: la estimulación temprana a pacientes prematuros, neuro rehabilitación infantil, rehabilitación en oncología mamaria, entre otras; ampliando así la oferta de servicios y el tipo de pacientes que se puedan beneficiar de la kinesiología en HD.

\section{CONCLUSIÓN Y PROYECCIONES}

Actualmente, no todos los hospitales del sistema de salud público chileno cuenta con unidad de hospitalización domiciliaria, y de los que sí tienen, no todos cuentan con kinesiología. Sin embargo, dado lo anterior expuesto, se evidencia que el kinesiólogo/a permite entregar diferentes prestaciones en domicilio tanto a adultos como niños y que debe estar presente como pilar fundamental en 
el equipo de HD, con un perfil único y definido a nivel nacional, el cual pueda ser adaptado según las necesidades de cada unidad, enfocado principalmente en las tres áreas principales de la kinesiología (respiratoria, motora y neurológica) pudiendo tener la capacidad de innovar y crear nuevas prestaciones. Además, consideramos que la autonomía tanto en gestión como en el ámbito clínico/ asistencial del kinesiólogo es primordial siendo el único camino para que los pacientes reciban de manera adecuada su terapia. Finalmente, esperamos que a partir de los datos recolectados se pueda avanzar hacía lineamientos que permitan estandarizar la incorporación de los kinesiólogos/as a todas las unidades de hospitalización a nivel nacional y en un futuro a nivel internacional.

\section{BIBLIOGRAFÍA}

1. López Perona F. ¿Qué es la unidad de hospitalización domiciliaria? y la introducción a su gestión. Enferm Glob. 2003;2(2):1-8. DOI: 10.6018/eglobal.2.2.624

2. Fernández-Miera MF. Hospitalización a domicilio del anciano con enfermedad aguda. Rev Esp Geriatr Gerontol. 2009;44(S1):S39-S50. DOI: 10.1016/j.regg.2009.03.012; PMID: 19501428

3. Ortega Palavecinos M. Descripción del modelo de hospitalización domiciliaria de un hospital público de Chile [Tesis de Grado]. Santiago de Chile, Chile: Escuela de Gobierno y Gestión Pública, Universidad Academia de Humanismo Cristiano; 2018.

4. Pavlovic A, Calderón Ó, Muñoz E, Cárcamo M, Trian J, Morales K. Descripción de los pacientes mayores de 60 años ingresados al programa de hospitalización domiciliaria del complejo asistencial Dr. Sótero del Río, Santiago de Chile. Acta Med Colomb. 2016;41(3):187-90.

5. Shepperd S, lliffe S. Hospital domiciliario versus atención hospitalaria estándar (Revisión Cochrane traducida) [monografía en Internet]. Barcelona, España: La Biblioteca Cochrane Plus del Centro Cochrane Iberoamericano; 2008 [accedido el 24 sep 2019]; Disponible en: http:// bit.ly/2KOLT54

6. Mitre Cotta RM, Suárez-Varela MM, Llopis González A, Cotta Filho JS, Real ER, Dias Ricós JA. La hospitalización domiciliaria: antecedentes, situación actual y perspectivas. Rev Panam Salud Pública. 2001;10(1):45-55. DOI: 10.1590/s1020-49892001000700007; PMID: 11558249

7. Cuxart Mèlich A, Estrada Cuxart O. Hospitalización a domicilio: oportunidad para el cambio. Med Clin (Barc). 2012;138(8):355-60. DOI: 10.1016/j.medcli.2011.04.008; PMID: 2169678

8. Levine DM, Ouchi K, Blanchfield B, Saenz A, Burke K., Paz M, et al. Hospital-Level Care at Home for Acutely III Adults: A Randomized Controlled Trial. Ann Intern Med. 2020; 172(2):77-85. DOI:10.7326/M19-0600; PMID: 31842232

9. Fonseca Figueroa J. Caracterización de los usuarios y de las prestaciones de la unidad de hospitalización domiciliaria del hospital San Juan de Dios, desde octubre 2010 hasta junio 2014 [Tesis Doctoral]. Santiago: Facultad de Medicina, Escuela de Salud Pública Universidad de Chile; 2015.

10. Freire Figueroa F, Marín Navarro V. Descripción e impacto económico de los pacientes con patologías respiratorias ingresados con indicación de kinesiología en la Unidad de Hospitalización Domiciliaria del Hospital Dr. Sótero del Río. Hosp Domic. 2019;3(4):243-54. DOI: 10.22585/ hospdomic.v3i4.86

11. Puchi Gómez, C, Sanhueza Alvarado O. Rol del profesional de enfermería en unidades de hospitalización domiciliaria de hospitales públicos. Rev Iberoam Educ Investi Enferm. 2015; 5(1):8-17. 
12. Poblete Figueroa C, Lagos Vásquez D, Marín Navarro V, Freire Figueroa F. Rol e Impacto del Kinesiólogo en la Unidad de Hospitalización Domiciliaria del Complejo Asistencia Dr. Sótero del Río. Kinesiología. 2018:37(3); 62.

13. Yorschua Jalil C, Gregory Villarroel S, Gonzalo Moscoso A, Patricio Barañao G, Méndez RM. Rol del kinesiólogo respiratorio en el proceso de alta domiciliaria en niños con ventilación mecánica prolongada. Neumol Pediatr. 2017; 12(4):161-8.

14. Rasmussen RS, Ostergaard A, Kjær P, Skerris A, Skou C, Christoffersen J, Overgaard, K. Stroke rehabilitation at home before and after discharge reduced disability and improved quality of life: A randomised controlled trial. Clin Rehabil. 2016; 30(3):225-36. DOI: 10.1177/0269215515575165; PMID: 25758941

15. Chaiyawat $P$, Kulkantrakorn $K$. Effectiveness of home rehabilitation program for ischemic stroke upon disability and quality of life: A randomized controlled trial. Clin Neurol Neurosurg. 2012; 114(7):866-70. DOI: 10.1016/j.clineuro.2012.01.018; PMID: 22321758

16. Paredes $\mathrm{O}$, Nuñez R, Klaber I. Successful initial experience with a novel outpatient total hip arthroplasty program in a public health system in Chile. Int Orthop. 2018; 42(8):1783-7. DOI: 10.1007/s00264-018-3870-6; PMID: 29564492

17. Ruiz-Sada P, Esquillor-Rodrigo M J, Palacios-García L, Iguzquiza-Pellejero M J, Oroz-Segura I, ResanoGarcía I. Estudio comparativo entre pacientes EPOC ingresados en hospitalización a domicilio y hospitalización convencional. Hosp Domic. 2017;1(4):189-97. DOI: 10.22585/hospdomic.v1i4.22

18. Hernández-Velásquez GM, Mosquera-Gómez LE, Pinzón-Rondón AM, Escobar JS, GuzmánPrado Y. Hospitalización domiciliaria pediátrica en el Instituto de Ortopedia Infantil Roosevelt en Bogotá, Colombia: enero a julio, 2013. Med UIS. 2016; 29(3):43-8. 